\title{
Simultaneous Conduction and Valence Band Quantization in Ultrashallow High-Density Doping Profiles in Semiconductors
}

\author{
F. Mazzola, ${ }^{1}$ J. W. Wells, ${ }^{1,}$ A. C. Pakpour-Tabrizi, ${ }^{2}$ R. B. Jackman, ${ }^{2}$ B. Thiagarajan, ${ }^{3}$ Ph. Hofmann, ${ }^{4}$ and J. A. Miwa ${ }^{4}$ \\ ${ }^{1}$ Center for Quantum Spintronics, Department of Physics, Norwegian University of Science and Technology, \\ NO-7491 Trondheim, Norway \\ ${ }^{2}$ London Centre for Nanotechnology and Department of Electronic and Electrical Engineering, University College London, \\ 17-19 Gordon Street, London WC1H OAH, United Kingdom \\ ${ }^{3}$ MAX-lab, P.O. Box 118, S-22100 Lund, Sweden \\ ${ }^{4}$ Department of Physics and Astronomy and Interdisciplinary Nanoscience Center (iNANO), Aarhus University, \\ Ny Munkegade 120, DK-8000 Aarhus, Denmark
}

(Received 22 November 2016; revised manuscript received 8 December 2017; published 26 January 2018)

\begin{abstract}
We demonstrate simultaneous quantization of conduction band (CB) and valence band (VB) states in silicon using ultrashallow, high-density, phosphorus doping profiles (so-called Si:P $\delta$ layers). We show that, in addition to the well-known quantization of CB states within the dopant plane, the confinement of VB-derived states between the subsurface P dopant layer and the Si surface gives rise to a simultaneous quantization of VB states in this narrow region. We also show that the VB quantization can be explained using a simple particle-in-a-box model, and that the number and energy separation of the quantized VB states depend on the depth of the P dopant layer beneath the Si surface. Since the quantized CB states do not show a strong dependence on the dopant depth (but rather on the dopant density), it is straightforward to exhibit control over the properties of the quantized CB and VB states independently of each other by choosing the dopant density and depth accordingly, thus offering new possibilities for engineering quantum matter.
\end{abstract}

DOI: $10.1103 /$ PhysRevLett.120.046403

There has been a surge of interest in two-dimensional (2D) materials due to their remarkable quantum properties. Graphene and layered transition metal dichalcogenides are just two examples of such materials that have been recently studied and proffered as advantageous for developing quantum electronic devices [1-4]. A rather unique branch of the 2D material family are ultrashallow, high-density, doping profiles in semiconductors, so-called $\delta$ layers. In particular, phosphorus $\delta$ layers in silicon (Si:P $\delta$ layers) combined with atomically precise lithography have led to recent technological successes towards scalable qubit architectures [5-8]. It has been demonstrated that $\mathrm{P}$ donors, which can act as qubits, in Si have long spin lifetimes $[9,10]$ which are essential for spin-based quantum calculations. Importantly, Si:P $\delta$ layers can be readily synthesized-they are composed of a $\mathrm{Si}(001)$ substrate with a high-density $\mathrm{P}$ dopant profile situated a few nanometers beneath an epitaxial grown $\mathrm{Si}$ encapsulation layer-and they are potentially straightforward to integrate into existing $\mathrm{Si}$ based technology. Both the dopant layer and the encapsulation layer can be easily modified during the growth process [11-13], and it is this flexibility that makes $\delta$ layers so promising, not only for enhancing the performance of quantum electronic devices, but for engineering new 2D materials with new capabilities.

The confinement of a high-density, atomically thin layer of $\mathrm{P}$ atoms beneath the surface abruptly changes the potential within the Si crystal. This brings about strong bending of the conduction band (CB) and valence band (VB) around the dopant plane, leading to strong confinement of the silicon CB. This strong confinement results in lowering and discretization of the $\mathrm{CB}$ and consequently gives the system metallic character [14-18]. These CB states have been studied in considerable detail [16,19-21], and it has been determined that their binding energy and energy separation (so-called valley splitting) can be effectively controlled and tuned by varying the $\mathrm{P}$ doping density and/or depth profile [22].

It is not only essential for device operation and performance that the quantized $\mathrm{CB}$ states can be tuned and controlled but also their VB counterparts. We demonstrate a general method based on $\delta$ doping to realize simultaneous quantization of $\mathrm{CB}$ and $\mathrm{VB}$ electrons by structuring the band bending at the nanoscale. We show, using angleresolved photoemission spectroscopy (ARPES), that quantized VB states arise from confinement between the $\mathrm{P}$ dopant layer and surface of the Si encapsulation. We verify that these quantized VB states can be tuned by varying the thickness of the Si encapsulation. This capability promises new prospects in engineering quantum matter, for example, the possibility of controlling carrier lifetimes by modifying the interaction between quantized CB and VB states.

ARPES measurements were performed at the I4 beam line at the MAX-III synchrotron radiation source [23]. The 
energy and momentum resolutions were better than $40 \mathrm{meV}$ and $0.02 \AA^{-1}$, respectively. The base pressure in the analysis chamber was $\approx 5 \times 10^{-10} \mathrm{mbar}$, and the temperature of the sample was maintained at room temperature throughout data acquisition. The Si:P $\delta$-layer samples were prepared by growing epitaxial $\mathrm{Si}$ (thicknesses from 1 to $4 \mathrm{~nm}$ ) on top of $\approx 0.25$ monolayers of $\mathrm{P}$ atoms incorporated in the topmost layer of a clean $\mathrm{Si}(001)$ substrate; a detailed recipe can be found in Ref. [16]. The arrangement of incorporated P atoms in the Si substrate has been investigated by combined atomresolved scanning tunneling microscopy [24] and density functional theory [25]. The results of these studies suggest the incorporated $\mathrm{P}$ atoms exhibit some short-range but no long-range ordering. Control samples were measured for comparison and fabricated by growing a similar amount of epitaxial Si directly on the clean $\mathrm{Si}(001)$ substrate without the inclusion of a P-rich layer.

The ARPES acquisitions of a control sample and a Si:P $\delta$-layer sample with an approximate 4-nm-thick Si encapsulation layer are presented in Figs. 1(a) and 1(b), respectively. The two samples have similar spectral features: the VB dispersion around the $\bar{\Gamma}$ point of the 2D Brillouin zone and surface states (SS) are consistent with bulk and surface states previously reported for electronic structure measurements of $\mathrm{Si}(001)$ with a $2 \times 1$ reconstructed surface [26,27].

The Fermi level $\left(E_{F}\right)$ lies within the $1.1 \mathrm{eV}$ band gap and is situated in close proximity to the conduction band minimum, confirming the $n$-type doping. There are notable differences between the two samples: an additional feature near $E_{F}$ and extra bands in the VB region-marked by the black rectangles - can be seen in the ARPES data of the Si: $\mathrm{P} \delta$-layer sample shown in Fig. 1(b). These differences are

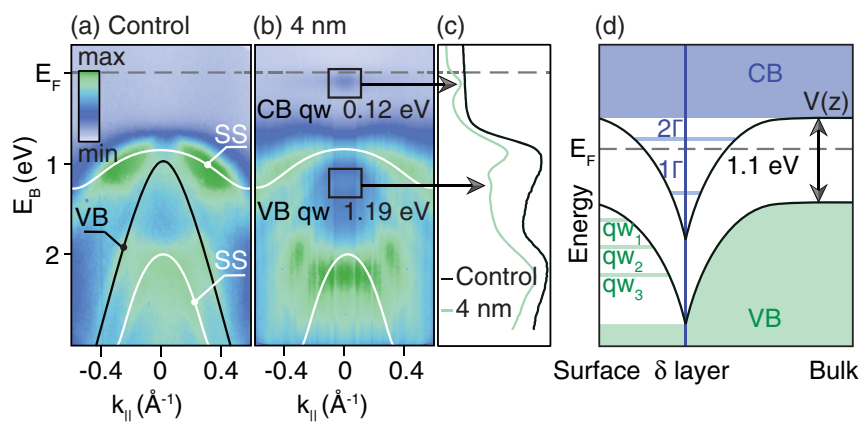

FIG. 1. Simultaneous quantization for $\mathrm{CB}$ and VB states in silicon. (a) ARPES data for the control sample, (b) corresponding ARPES data of a Si:P $\delta$-layer sample with a $4 \mathrm{~nm}$ encapsulation thickness, CB and VB states indicated. (c) Momentum integrated EDCs to emphasize the differences between the two samples. (d) Band bending schematic of a Si:P $\delta$ layer. The resulting potential, $V(z)$, is shown by the black line and confines the $\mathrm{CB}$ band electrons to give rise to the states labeled $1 \Gamma$ and $2 \Gamma$. Recovery of the potential well to the surface leads to quantized states confined to the Si encapsulation layer: $q w_{1}, q w_{2}$, and $q w_{3}$. The blue and green shaded areas represent the continuum of $\mathrm{CB}$ and VB bulk states where these quantized states cannot form. prominent in Fig. 1(c) where energy distribution curves (EDCs), integrated over a momentum range of -0.15 to $0.15 \AA^{-1}$, are plotted. The additional states appear as peaks at binding energies of 0.12 and $1.19 \mathrm{eV}$ for the Si:P $\delta$-layer sample (green curve) and are noticeably absent in the control sample (black curve).

We use the band bending diagram of a Si:P $\delta$ layer in Fig. 1(d) to illustrate the origin of these additional states. As we go from bulk to surface, i.e., from right to left across the diagram, the bulk $\mathrm{CB}$ becomes partially occupied in the region around the high-density $\mathrm{P}$ dopant plane, thereby creating a confined metallic layer. The $\mathrm{CB}$ states which are bound by the Coulomb-like potential well are labeled $1 \Gamma$ and $2 \Gamma$. While these electronic states have already been studied in detail by ARPES [19], an understanding of the nature and origin of the extra bands that are visible in the VB region is lacking. Previous ARPES measurements have shown that both the $\mathrm{CB}$ and VB states are nondispersing with photon energy, firmly establishing the 2D character of these coexisting states [16]. ARPES acquisitions at photon energy of $36 \mathrm{eV}$ are only presented here, as the intensity of the CB states is known to be enhanced at this energy [16,21].

If VB states should exist between the surface and the $\delta$ layer, they too must be strongly confined since both the surface and the $\delta$ layer act as a barrier [see left-hand side of Fig. 1(d)]. Therefore, the holelike bands of the VB become trapped like a particle in a box, where the confinement width is dictated by the depth of the $\delta$ layer beneath the surface and the confinement potential is dictated by the Fermi level pinning at the surface and at the $\delta$ layer. All of these parameters can be controlled during the sample growth.

We have explored the influence of $\mathrm{Si}$ encapsulation layer thickness on the quantization of $\mathrm{CB}$ and VB states using ARPES. First of all, we want to study the thickness dependent photoemission intensity, as this will give information about whether a certain electronic state is bulk or surface derived. In Figs. 2(b)-2(e) we consider the following Si encapsulation thicknesses: 1, 2, 3, $4 \mathrm{~nm}$ and compare them with the ARPES measurements for the control sample shown in Fig. 2(a). At first glance, all of the ARPES spectra of the different Si:P $\delta$-layer samples appear qualitatively similar to each other. A pronounced difference is the diminishing spectral weight of $1 \Gamma$ near the $E_{F}$ for Si:P $\delta$ layers with thicker encapsulation. Since $1 \Gamma$ originates from the $\mathrm{P}$ dopant layer situated beneath the surface, it is expected that the signal intensity gets weaker for thicker $\mathrm{Si}$ encapsulations. In Fig. 2(f), EDCs (integrated over a momentum range of -0.15 to $0.15 \AA^{-1}$ ) are plotted for the control sample and the four Si:P $\delta$ layers. In this manner, the peak intensities of both the CB and VB quantized states, marked by the arrows, can be directly compared. For increasing Si encapsulation thicknesses, a decrease in the intensity of the quantized $\mathrm{CB}$ peak corresponds to an increase in the intensity of the quantized VB states. This is 


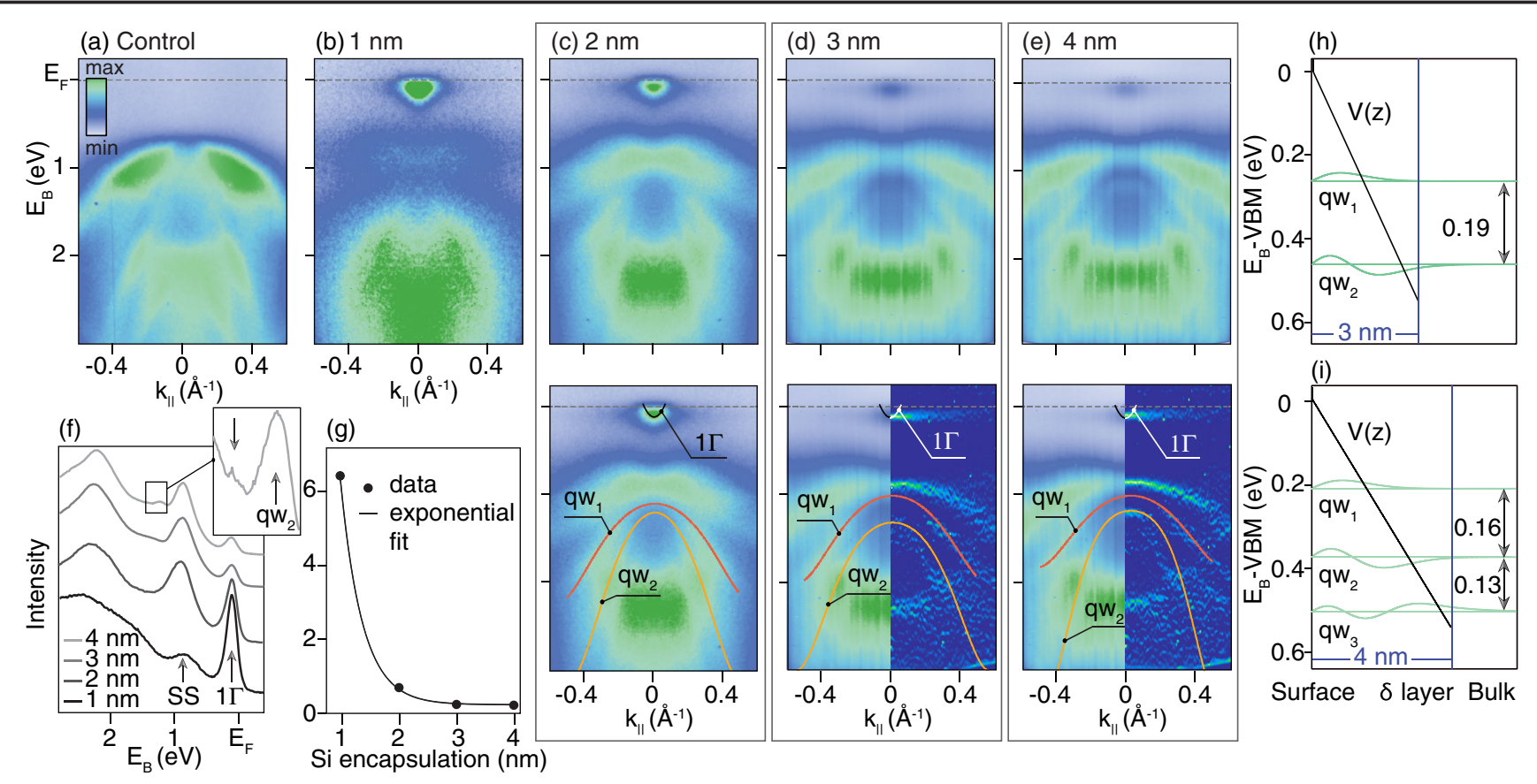

FIG. 2. Evolution of the quantized VB states with encapsulation thickness. (a) ARPES data for the control Si sample. (b)-(e) Si:P $\delta$-layer ARPES spectra acquired for different Si encapsulation thicknesses ranging from 1 to $4 \mathrm{~nm}$. The $\mathrm{CB}$ state at the Fermi level (1Г) becomes gradually weaker with increasing Si encapsulation thickness while the states within the VB region become more intense. For panels (c)-(e), the ARPES spectra are shown twice with salient features marked and labeled on the spectra displayed in the lower panels. To enhance the visibility of the quantized VB states, the curvature method [28] was applied and the results are presented on right-hand sides of the lower panels of (d) and (e). The spectra for the 1-4 nm encapsulation thicknesses have been left-right symmetrized, while the control sample has not. An even sixth-order polynomial was used to fit the quantized VB states, $q w_{1}$ (orange) and $q w_{2}$ (yellow), for the data shown in (c)-(e) [29]. (f) Momentum integrated EDCs for Si:P $\delta$ layers with different encapsulation thicknesses. The positions of the CB $(1 \Gamma)$ and SS are indicated. The inset shows an enlarged region of the 4-nm-thick Si encapsulation data where the $q w_{2}$ state is readily visible. Adjacent to the $q w_{2}$ state, an unlabeled arrow marks the location of small peak which may be due to a third quantized valence band state. (g) The intensity ratio of CB to VB states for each of the $\mathrm{Si}: \mathrm{P} \delta$-layer samples. (h), (i) Numerically obtained solutions to the Schrödinger equation for a linear potential well $[V(z)$, black line] for 3 and $4 \mathrm{~nm}$ Si encapsulation layers, respectively. The calculated eigenstates are marked by the green curves and the separation energies (in eV) marked by the double-headed arrows.

confirmed in Fig. 2(g) where the spectral intensity of the $\mathrm{CB}$ is plotted (relative to the intensity of the VB states, i.e., $\left.I_{\delta} / I_{\mathrm{VB}}\right)$, as a function of $\mathrm{Si}$ encapsulation thickness, and shows an exponential suppression for photoelectrons emitted from deeper $\mathrm{P}$ dopant layers. While the subsurface origin of the quantized CB states was known, this analysis suggests that the quantized VB states exist up to the surface.

By increasing the thickness of the encapsulation from 3 to $4 \mathrm{~nm}$, the energy separation between the quantized VB states decreases; compare $q w_{1}$ (orange) and $q w_{2}$ (yellow) in Figs. 2(d) and 2(e). This trend can be explained by a particle-in-a-box picture: as the width of the box, or in this case the thickness of the $\mathrm{Si}$ encapsulation, is increased, the energies of the quantum states are lowered. We note that the energy separation between the quantized VB states for the $2 \mathrm{~nm}$ encapsulation thickness [Fig. 2(c)] does not follow this trend. This exception may be due to the complex interaction of the SS, located at $E_{b} \approx 1 \mathrm{eV}$, with the quantized VB states. While the physical extent of the SS wave function is relatively shallow [30], a broadening and shifting could still be expected for a sufficiently small spatial separation of the SS and the quantized VB states.

The dispersion of the quantized VB states was fitted using an even sixth-order polynomial (orange and yellow curves in Fig. 2), and their effective masses and uncertainties estimated [31]. We expect the different encapsulation thicknesses to have a small effect on the effective masses of the quantised VB states. Given the associated uncertainties, the effective masses for the $q w_{1}$ state are in agreement with the heavy-hole state in the bulk VB of Si. The effective masses for the $q w_{2}$ state are less, but also probably derived from the bulk heavy holes since their effective masses are more similar to that of the bulk heavyhole state than the bulk light-hole state.

Additional confirmation that the extra features in the VB region are quantized $\mathrm{VB}$ states confined within the $\mathrm{Si}$ encapsulation layer is provided by our numerical model for solving the Schrödinger equation presented in Figs. 2(h) and 2(i). For simplicity we only consider a linear potential $[V(z)$, black line $]$ between the dopant layer and the surface. The approximation is crude but reproduces the quantized 
VB states seen in the ARPES measurements of Fig. 2. It is worth noting that we only apply our model to $\mathrm{Si}: \mathrm{P} \delta$ layers with the thickest encapsulation layers studied here, i.e., 3 and $4 \mathrm{~nm}$, since the quantized VB states and the surface states are well separated for these cases, thereby facilitating the comparison between data and model. The interaction of the SS with the quantized VB states, for the 1 and $2 \mathrm{~nm}$ cases, is simply not captured in this model that assumes a quantum well with the same boundary conditions for every thickness.

In our model, by increasing the thickness from 3 to $4 \mathrm{~nm}$, the number of solutions to the Schrödinger equation increases from two to three. For the $3 \mathrm{~nm}$ case, the two calculated states are assigned to the $q w_{1}$ and $q w_{2}$ states observed in the experiment; see Fig. 2(d). The experimental data in Fig. 2(e) show a weak hint of a third $q w_{3}$ state expected for the $4 \mathrm{~nm}$ case, but the intensity of the signal is weak and comparable to the background. The reduced intensity of the state may also be a result of its wave function being less localized at the surface (compared to $q w_{1}$ and $\left.q w_{2}\right)$ as illustrated in Fig. 2(i), or due to the fact that the photoionization cross section of this state is lower at this photon energy [16] (or, most likely, both effects might play a role).

We extracted EDCs, integrated over a finite momentum range, for $\mathrm{Si}: \mathrm{P} \delta$ layers with different encapsulation thicknesses to investigate further this possible $q w_{3}$ state. In the inset of Fig. 2(f), the $q w_{2}$ state is readily visible for the 4-nm-thick Si encapsulation data, and adjacent to this state, there is a small peak where the $q w_{3}$ state may be expected.

The energy separations between the quantized VB states are determined from the numerical model to be $q w_{2}-q w_{1}=0.19 \mathrm{eV}$ for the 3-nm-thick Si encapsulation, and $q w_{2}-q w_{1}=0.16 \mathrm{eV}$ and $q w_{3}-q w_{2}=0.13 \mathrm{eV}$ for the 4-nm-thick layer. From the experimental data we measure an energy separation between the two lowest lying states to be $q w_{2}-q w_{1}=0.30 \pm 0.17 \mathrm{eV}$ for the 3-nm-thick Si encapsulation and $q w_{2}-q w_{1}=0.17 \pm 0.12 \mathrm{eV}$ for the 4-nmthick layer, respectively $\left(3 \mathrm{~nm}, q w_{1}=1.02 \pm 0.08 \mathrm{eV}\right.$ and $q w_{2}=1.32 \pm 0.09 \mathrm{eV} ; 4 \mathrm{~nm}, q w_{1}=1.02 \pm 0.08 \mathrm{eV}$ and $\left.q w_{2}=1.19 \pm 0.04 \mathrm{eV}\right)$. The experimental values for all the quantized VB states are different from the ones extracted numerically; however, the general trend holds for the thicker encapsulation thicknesses: (i) a shift of the quantized states toward lower binding energy and (ii) a decrease in the energy separation between higher to lower lying states for increasing Si encapsulation thickness is observed. This supports the notion that the quantized VB states originate from confinement in the Si encapsulation layer. We expect that a more accurate model for the doping potential and its recovery to the surface, including the influence of the SS wave function, might be able to give more realistic energy separations.

Simultaneous quantization of CB and VB has not been demonstrated in common semiconductors; previously, a special case of simultaneous quantization of the $\mathrm{CB}$ and VB has been reported for the topological insulator $\mathrm{Bi}_{2} \mathrm{Se}_{3}$ [32]. The adsorption of $\mathrm{CO}$ gas on the $\mathrm{Bi}_{2} \mathrm{Se}_{3}$ surface induces a similar downward band bending of the $\mathrm{CB}$ and the formation of quantized $\mathrm{CB}$ states. However, the quanitzed VB has quite another origin: $\mathrm{Bi}_{2} \mathrm{Se}_{3}$ has a peculiar valence electronic structure near the center of its surface Brillouin zone-in this region the upper VB only exists in a narrow ( $\approx 200 \mathrm{meV}$ ) energy window-and thus downward bending of the VB can also lead to quantized states. The origin of the $\mathrm{CB}$ and VB quantization is completely different in a $\delta$ layer since the simultaneous quantization of the $\mathrm{CB}$ and $\mathrm{VB}$ is purely artificial: it is dictated by the type, density, and profile of the dopant layer and unlike $\mathrm{Bi}_{2} \mathrm{Se}_{3}$ is not an innate and unusual property of the bulk material. Artificially induced quantization of the $\mathrm{CB}$ and VB by $\delta$ doping offers the realization of the same effect in a wide spectrum of semiconductor hosts.

The properties of the band bending in $\delta$ layers can be easily modified during the growth process, and as a result quantization of the $\mathrm{CB}$ and VB can be controlled and tuned. We can, for example, also occupy the $2 \Gamma$ state so that it is situated below the $E_{F}[19,22]$, by either increasing the P dopant density or broadening the $\mathrm{P}$ dopant profile of the $\delta$ layer. The surface of the $\mathrm{Si}$ encapsulation layer will similarly impact the quantization of the VB states as different surface terminations or surface adsorbates can alter the $E_{F}$ pinning at the surface, and thus modify the degree of band bending between the dopant layer and the surface.

The situation of simultaneous quantization of electron and hole states is a rather unusual effect [32], never observed before in traditional doped semiconductors. This effect provides the appealing prospect of controlling the lifetime of carriers, creating additional channels to generate electron-hole pair recombinations in the $\mathrm{CB}$, mediated by electronic transitions from the VB, potentially controlled by a biased top gate (analogous to a field effect transistor): that is, to mediate transitions between the $\mathrm{CB}$ and VB by tuning the potential landscape in which these states reside by modification of the dopant layer and the surface termination. For example, surface doping would directly influence the barrier potential responsible for the near-surface quantized VB states, and thus directly influence their energy, but would have a minimal influence on the subsurface quantized CB (and bulk VB), for which the $\delta$ layer and bulk doping densities, respectively, determine the Fermi level pinning. Thus, by modifying the surface potential, it should be possible to deliberately align (or misalign) the energies of the quanitized VB and CB states so as to exhibit control of their interaction (and, therefore, lifetime). The flexibility that these $\delta$ layers offer could be expected to play a major role in the performance of quantum electronic devices.

In summary, simultaneous quantization of the $\mathrm{CB}$ and VB states of Si:P $\delta$ layers has been experimentally verified using ARPES. The origins of these quantized states are 
different: the $\mathrm{CB}$ states arise from the potential well induced by the ultradense dopant layer whereas the VB states originate from confinement between the potential well created by the dopant layer and the sample surface. All of the relevant properties of both the dopant and encapsulation layers can be easily controlled and modified during the $\delta$-layer growth process, not only providing the ability to exhibit control of the quantization of CB and VB states, but also offering the intriguing possibility of influencing lifetimes within the $\delta$-layer structure, thereby opening up new possibilities for engineering quantum materials with new capabilities.

We acknowledge Johan Adell for support at the I4 beam line at MAX-III. Partial funding for this work was obtained through the Norwegian Ph.D. Network on Nanotechnology for Microsystems sponsored by the Research Council of Norway, Division for Science under Contract No. 221860/ F40. J. A. M. acknowledges support from the Danish Council for Independent Research, Natural Sciences under the Sapere Aude program (Grant No. DFF-6108-00409) and the Aarhus University Research Foundation. This work was supported by VILLUM FONDEN via the Centre of Excellence for Dirac Materials (Grant No. 11744) and partly supported by the Research Council of Norway through its Centres of Excellence funding scheme, Project No. 262633, "QuSpin," and through the Fripro program, Project No. 250985 "FunTopoMat."

*quantum.wells@gmail.com

[1] K. S. Novoselov, A. K. Geim, S. V. Morozov, D. Jiang, Y. Zhang, S. V. Dubonos, I. V. Grigorieva, and A. A. Firsov, Science 306, 666 (2004).

[2] K. S. Novoselov, Z. Jiang, Y. Zhang, S. V. Morozov, H. L. Stormer, U. Zeitler, J. C. Maan, G. S. Boebinger, P. Kim, and A. K. Geim, Science 315, 1379 (2007).

[3] K. F. Mak, K. L. McGill, J. Park, and P. L. McEuen, Science 344, 1489 (2014).

[4] X. Xu, W. Yao, D. Xiao, and T. F. Heinz, Nat. Phys. 10, 343 (2014).

[5] M. Fuechsle, J. A. Miwa, S. Mahapatra, H. Ryu, S. Lee, O. Warschkow, L. C. L. Hollenberg, G. Klimeck, and M. Y. Simmons, Nat. Nanotechnol. 7, 242 (2012).

[6] B. Weber, S. Mahapatra, H. Ryu, S. Lee, A. Fuhrer, T. C. G. Reusch, D. L. Thompson, W. C. T. Lee, G. Klimeck, L. C. L. Hollenberg et al., Science 335, 64 (2012).

[7] C. D. Hill, E. Peretz, S. J. Hile, M. G. House, M. Fuechsle, S. Rogge, M. Y. Simmons, and L. C. L. Hollenberg, Sci. Adv. 1, e1500707 (2015).

[8] S. R. McKibbin, G. Scappucci, W. Pok, and M. Y. Simmons, Nanotechnology 24, 045303 (2013).

[9] T. Suzuki, T. Sasaki, T. Oikawa, M. Shiraishi, Y. Suzuki, and K. Noguchi, Appl. Phys. Express 4, 023003 (2011).
[10] G. Wolfowicz, A. M. Tyryshkin, R. E. George, H. Riemann, N. V. Abrosimov, P. Becker, H.-J. Pohl, M. L. W. Thewalt, S. A. Lyon, and J. J. L. Morton, Nat. Nanotechnol. 8, 561 (2013).

[11] K. E. J. Goh, L. Oberbeck, M. Y. Simmons, A. R. Hamilton, and R. G. Clark, Appl. Phys. Lett. 85, 4953 (2004).

[12] K. E. J. Goh, L. Oberbeck, M. Y. Simmons, A. R. Hamilton, and M. J. Butcher, Phys. Rev. B 73, 035401 (2006).

[13] K. E. J. Goh and M. Y. Simmons, Appl. Phys. Lett. 95, 142104 (2009).

[14] D. J. Carter, O. Warschkow, N. A. Marks, and D. R. McKenzie, Phys. Rev. B 79, 033204 (2009).

[15] D. J. Carter, N. A. Marks, O. Warschkow, and D. R. McKenzie, Nanotechnology 22, 065701 (2011).

[16] J. A. Miwa, P. Hofmann, M. Y. Simmons, and J. W. Wells, Phys. Rev. Lett. 110, 136801 (2013).

[17] C. M. Polley, W. R. Clarke, J. A. Miwa, M. Y. Simmons, and J. W. Wells, Appl. Phys. Lett. 101, 262105 (2012).

[18] C. M. Polley, W. R. Clarke, J. A. Miwa, G. Scappucci, J. W. Wells, D. L. Jaeger, M. R. Bischof, R. F. Reidy, B. P. Gorman, and M. Simmons, ACS Nano 7, 5499 (2013).

[19] J. A. Miwa, O. Warschkow, D. J. Carter, N. A. Marks, F. Mazzola, M. Y. Simmons, and J. W. Wells, Nano Lett. 14, 1515 (2014).

[20] F. Mazzola, C. M. Polley, J. A. Miwa, M. Y. Simmons, and J. W. Wells, Appl. Phys. Lett. 104, 173108 (2014).

[21] F. Mazzola, M. T. Edmonds, K. Høydalsvik, D. J. Carter, N. A. Marks, B. C. C. Cowie, L. Thomsen, J. Miwa, M. Y. Simmons, and J. W. Wells, ACS Nano 8, 10223 (2014).

[22] F. Mazzola, J. A. Miwa, R. Rahman, X.-G. Zhu, M. Simmons, P. Hofmann, and J. Wells (to be published).

[23] B. Jensen, S. Butorin, T. Kaurila, R. Nyholm, and L. Johansson, Nucl. Instrum. Methods Phys. Res., Sect. A 394, 243 (1997).

[24] N. J. Curson, S. R. Schofield, M. Y. Simmons, L. Oberbeck, J. L. O'Brien, and R. G. Clark, Phys. Rev. B 69, 195303 (2004).

[25] H. F. Wilson, O. Warschkow, N. A. Marks, N. J. Curson, S. R. Schofield, T. C. G. Reusch, M. W. Radny, P. V. Smith, D. R. McKenzie, and M. Y. Simmons, Phys. Rev. B 74, 195310 (2006).

[26] L. S. O. Johansson, P. E. S. Persson, U. O. Karlsson, and R. I. G. Uhrberg, Phys. Rev. B 42, 8991 (1990).

[27] L. S. O. Johansson, R. I. G. Uhrberg, P. Mårtensson, and G. V. Hansson, Phys. Rev. B 42, 1305 (1990).

[28] P. Zhang, P. Richard, T. Qian, Y.-M. Xu, X. Dai, and H. Ding, Rev. Sci. Instrum. 82, 043712 (2011).

[29] See Supplemental Material at http://link.aps.org/ supplemental/10.1103/PhysRevLett.120.046403 for details regarding the fit analysis.

[30] M. Yengui, H. P. Pinto, J. Leszczynski, and D. Riedel, J. Phys. Condens. Matter 27, 045001 (2015).

[31] See Supplemental Material at http://link.aps.org/ supplemental/10.1103/PhysRevLett.120.046403 for details regarding the estimates of the effective masses.

[32] M. Bianchi, R. C. Hatch, J. Mi, B. B. Iversen, and P. Hofmann, Phys. Rev. Lett. 107, 086802 (2011). 